\title{
Gravitational waves from fundamental axion dynamics
}

\author{
Anish Ghoshal ${ }^{a, b}$ and Alberto Salvio ${ }^{a, c}$ \\ ${ }^{a}$ I.N.F.N. - Rome Tor Vergata, \\ via della Ricerca Scientifica, I-00133 Rome, Italy \\ ${ }^{b}$ I.N.F.N., Laboratori Nazionali di Frascati, \\ C.P. 13, I-00044 Frascati, Italy \\ ${ }^{c}$ Physics Department, University of Rome Tor Vergata, \\ via della Ricerca Scientifica, I-00133 Rome, Italy \\ E-mail: Anish.Ghoshal@roma2.infn.it, alberto.salvio@roma2.infn.it
}

ABSTRACT: A totally asymptotically free QCD axion model, where all couplings flow to zero in the infinite energy limit, was recently formulated. A very interesting feature of this fundamental theory is the ability to predict some low-energy observables, like the masses of the extra fermions and scalars. Here we find and investigate a region of the parameter space where the Peccei-Quinn (PQ) symmetry is broken quantum mechanically through the Coleman-Weinberg mechanism. This results in an even more predictive framework: the axion sector features only two independent parameters (the PQ symmetry breaking scale and the QCD gauge coupling). In particular, we show that the PQ phase transition is strongly first order and can produce gravitational waves within the reach of future detectors. The predictivity of the model leads to a rigid dependence of the phase transition (like its duration and the nucleation temperature) and the gravitational wave spectrum on the $\mathrm{PQ}$ symmetry breaking scale and the QCD gauge coupling.

Keywords: Beyond Standard Model, Cosmology of Theories beyond the SM, Renormalization Group

ARXIV EPRINT: 2007.00005 


\section{Contents}

1 Introduction 1

2 A fundamental axion model without a fundamental PQ scale 4

3 Quantum generation of $f_{a}$

4 Peccei-Quinn phase transition $\quad 8$

$\begin{array}{lll}5 & \text { Gravitational waves } & 14\end{array}$

6 Gravitational wave detectors $\quad 15$

$\begin{array}{llr}7 & \text { Conclusions } & 18\end{array}$

\section{Introduction}

The PQ symmetry [1] provides an elegant solution of the strong CP problem: it explains dynamically why the strong interactions preserve $\mathrm{CP}$, while the electroweak ones break it. This solution, regardless of its implementation, manifests itself at low energies through the axion, the pseudo-Goldstone boson associated with the breaking of this (approximate) symmetry [2]. Moreover, the axion is a good dark matter (DM) candidate and can actually account for the whole DM when the PQ symmetry breaking scale $f_{a}$ is around $10^{11} \mathrm{GeV}$ [3-5]. Even if there may be other components of DM, astrophysical observations require anyhow $f_{a}$ to be above the scale of $\sim 10^{8} \mathrm{GeV}$ (see ref. [6] for a recent review). This makes it difficult to test the PQ idea as current colliders are far from probing those energies.

Cosmology, on the other hand, allows us to have a window on physical processes occurring at such high mass scales. A classic example is the observation of the cosmic microwave background (CMB) anisotropies. Indeed, these give us information on the inflationary dynamics, which typically occurs at energies much above those within the reach of colliders (see refs. [7, 8] for up-to-date observations). Recently, the experimental discovery of gravitational waves (GWs) [9] has opened another window on very energetic processes predicted by particle physics models. An important example is the possibility to have experimental information on the characteristics of phase transitions, if these are strongly first order (see ref. [10] for a textbook introduction to this topic). Moreover, the possible observation of GWs due to a first-order phase transition would be a remarkably clear signal of new physics as the finite-temperature symmetry breaking dynamics in the Standard Model (SM) is not of this type. 
If the PQ phase transition is strongly first order it could lead to observable GWs. But, as pointed out in refs. [11, 12], the particular features of the phase transition and the corresponding GWs depend on the specific dynamics implementing the PQ symmetry. ${ }^{1}$ These features include, for example, the temperature at which the phase transition occurs, its duration and the GW spectrum.

One way one can significantly reduce this ambiguity, and thus have a guide regarding the directions towards which the experimental efforts may be focused, is considering only fundamental, that is truly UV complete, QCD axion models. This is because fundamental field theories, such as asymptotically free [17-20] or asymptotically safe [21, 22] models, have typically the ability to predict the value of some observables (see e.g. ref. [23]).

Recently, the first fully calculable and realistic QCD axion model of this sort has been constructed [24]. It features a new non-Abelian interaction and new quarks and scalars. This model is valid up to infinite energy because is totally asymptotically free (TAF): all couplings can flow to zero in the infinite energy limit. The requirement of total asymptotic freedom is a sufficient but non-necessary feature of a fundamental theory, as asymptotic safety can be an alternative (see [25-29] for phenomenological applications). However, having all couplings approach zero in the UV allows us to trust perturbation theory, at least for sufficiently high energies, and thus obtain a calculable model.

This asymptotically free axion model, as is typically the case in fundamental field theories, predicts some low-energy observables: this is because some couplings (specifically the Yukawa and quartic couplings) are compatible with the TAF requirement only if they acquire some specific isolated values at low energy. In other worlds, these couplings are IR attractive.

The requirement of having a classically scale-invariant axion sector, with no dimensionful parameters in the classical Lagrangian, further reduce the number of independent parameters. Indeed, the mass scales are then obtained quantum mechanically rather than with additional mass parameters in the classical Lagrangian. For this reason we investigate here a region of the parameter space of the TAF axion model [24] where the PQ symmetry is broken quantum mechanically through the Coleman-Weinberg (CW) mechanism [30]. This mechanism allows us, among other things, to generate scales via quantum corrections in the regime of validity of perturbation theory and, therefore, have a fully calculable setup.

The main purpose of this work is to study the PQ phase transition and the characteristics of the possible associated GWs in this highly predictive framework. As pointed out in ref. [31] (see [11, 12] for recent applications to QCD axion models) the phase transitions associated with the CW symmetry breaking are typically of first order and can, therefore, lead to observable GWs. ${ }^{2}$ This, together with the high predictivity of the TAF axion model mentioned above, can lead to testable implications at GW detectors. Furthermore, a typical feature of CW phase transitions is the presence of a phase of strong supercooling, meaning that the temperature below which the phase transition is effective (the nucleation

\footnotetext{
${ }^{1}$ See also refs. [13-16] for other studies of GWs in axion and axion-like effective models.

${ }^{2}$ See e.g. refs. [32-39] for previous studies of GWs and phase transitions in effective models with CW symmetry breaking.
} 
temperature) turns out to be much smaller than the critical temperature [31] and, for the PQ symmetry breaking, much below $f_{a}[11,12]$.

Since the TAF requirement regards the extrapolation of the theory at arbitrarily high energies, some comments about the behavior of gravity in the UV are now in order. Here we assume that the gravitational interactions are softened (compared to their behavior in Einstein's theory) above and only above a certain energy scale $\Lambda_{G}$ [23]. While at large lengths all successes of Einstein's theory are reproduced, gravity is assumed to be so weak from the UV down to the PQ scale that its impact on the renormalization group equations (RGEs) can be neglected. This is possible because $\Lambda_{G}$ can be much below the Planck scale $\bar{M}_{P}$, where quantum gravity effects in Einstein gravity would become sizeable. Since a phase of strong supercooling occurs in our classically scale-invariant TAF axion model, the nucleation temperature is much smaller than $f_{a}$ and the relevant values of the fields and their derivatives are also much smaller than $f_{a}$. This implies that gravity, as far as the production of gravitational waves is concerned, is well-described by Einstein's theory for all values of $\Lambda_{G}$ satisfying $f_{a} \lesssim \Lambda_{G} \ll \bar{M}_{P}$.

This softened-gravity scenario may be realized, for example, in UV modifications of gravity featuring quadratic curvature terms in the action [40-48] or in non-local extensions of general relativity [49-56]. Interestingly, the former case also admits a classically scaleinvariant formulation, called Agravity [40-43], in which the Planck and the Fermi scales as well as the cosmological constant are generated quantum mechanically via a gravitational version of the CW mechanism.

Given that gravity is softened for all energies above $\Lambda_{G}$ and Einstein's gravity is already very weak much below $\bar{M}_{P}$, in this scenario all gravitational contributions to the effective action can be well described by perturbation theory. This means that the non-perturbative Planckian corrections expected to spoil all global symmetries in Einstein's theory are actually negligible in the context of softened gravity. In particular the non-perturbative effects (described by Euclidean wormholes) that violate the PQ symmetry [57-60] and lead to the so-called PQ quality problem [61-66] in Einstein's gravity can be neglected here. Therefore, softened gravity provides automatically a solution of the PQ quality problem.

The paper is organized as follows. In section 2 we introduce the TAF axion model without a fundamental PQ scale and review results regarding the RG flow in [24] that are most relevant for our purposes. In section 3 it is shown that $f_{a}$ is generated quantum mechanically through the $\mathrm{CW}$ mechanism and the mass spectrum is also discussed. In the same section we also show that the axion sector only features one independent mass scale, i.e. $f_{a}$, and one independent dimensionless parameter (the QCD gauge coupling evaluated at $f_{a}$ ). The PQ phase transition is then studied in section 4 , which gives details about the finite-temperature effective potential, the bounce solutions and their actions, the nucleation temperature and the reheating after the supercooling era. In section 5 the predictions of the classically scale-invariant TAF axion model regarding the GW spectrum are worked out. A description of the relevant GW detectors is then provided in section 6 , where we also compare the sensitivities of the GW experiments with the predictions of our fundamental model. Finally, in section 7 we give our conclusions. 


\section{A fundamental axion model without a fundamental PQ scale}

Here we consider a dimensionless version of the TAF axion sector of [24]: we take the limit in which the dimensionful parameter in the microscopic Lagrangian go to zero. The axion sector is invariant under an $\mathrm{SU}(2)$ group (henceforth $\left.\mathrm{SU}(2)_{a}\right)$. Then the full gauge group contains the factor $\mathrm{SU}(3)_{c} \times \mathrm{SU}(2)_{a}$, where $\mathrm{SU}(3)_{c}$ is the ordinary QCD group. The gauge group should also include extra factors to account for a TAF extension of the SM (see e.g. [23, 67, 68]). We call such an extension the "SM sector", which of course has to be present, in addition to the axion sector we describe here in order for the complete model to be fully viable. We will not commit ourselves to a specific TAF SM extension in this work, but we note that in general the $\mathrm{SM}$ and axion sectors interact via $\mathrm{SU}(3)_{c}$ gauge interactions.

The model features two extra Weyl fermions $q$ and $\bar{q}$ in the fundamental and antifundamental of $\mathrm{SU}(3)_{c} \times \mathrm{SU}(2)_{a}$, with the same PQ charge: $\{q, \bar{q}\} \rightarrow e^{i \gamma / 2}\{q, \bar{q}\}$, where $\gamma$ is a constant. The PQ charges of all particles in the SM sector vanish for simplicity, like in axion models of the KSVZ [69-71] type. We introduce a scalar field $A$, which spontaneously breaks the PQ symmetry (denoted here $\mathrm{U}(1)_{\mathrm{PQ}}$ ) and gives mass to the extra quarks (as required by the experiments). Therefore, $A$ is complex and have Yukawa interactions with $q$ and $\bar{q}$,

$$
\mathscr{L}_{y}=-y \bar{q} A q+\text { H.c. . }
$$

The PQ symmetry implies that $A$ transforms under $\mathrm{U}(1)_{\mathrm{PQ}}$ as $A \rightarrow e^{-i \gamma} A$. Gauge invariance, instead, tells us that $A$ is invariant under $\mathrm{SU}(3)_{c}$ and belongs to the adjoint of $\mathrm{SU}(2)_{a}$. The scalar $A$, being complex, can be written as $A=A_{R}+i A_{I}$ where $A_{R}$ and $A_{I}$ are Hermitian adjoint representations. Further Yukawa interactions besides (2.1) and those present in the SM sector are forbidden by the gauge symmetries and $\mathrm{U}(1)_{\mathrm{PQ}}$.

The potential of $A$ is given by

$$
V_{A}=\lambda_{1} \operatorname{Tr}^{2}\left(A^{\dagger} A\right)+\lambda_{2}|\operatorname{Tr}(A A)|^{2} .
$$

Note that $V_{A}$, just like any other term in the Lagrangian, only contains dimensionless coefficients. The possible mass term $m^{2} \operatorname{Tr}\left(A^{\dagger} A\right)$ in the potential in [24] has been erased or, more generally speaking, it will be assumed that $m$ is much smaller than the effective mass generated by the CW mechanism (we will discuss how this mechanism works in the present model in section 3). The necessary and sufficient conditions for vacuum stability at high-field values (henceforth "high-field stability") are [24]

$$
\lambda_{1}>0, \quad \lambda_{1}+\lambda_{2}>0 .
$$

Here we neglect the couplings with the scalars of the SM sector; setting those couplings exactly to zero is consistent at one-loop level. At higher-loop level those couplings can be generated, but they remain small and will have negligible effects on our results.

Let us now review the beta-functions of this model [24]. The renormalization group equation (RGE) of the gauge coupling $g$ of a generic gauge group is

$$
\frac{d g^{2}}{d t}=-b g^{4}
$$


where $t \equiv \ln \left(\mu^{2} / \mu_{0}^{2}\right) /(4 \pi)^{2}$, the quantity $\mu_{0}$ is an arbitrary reference energy and $\mu$ is the usual RG scale. The solution to eq. (2.4) is UV attractive for any $g_{0} \equiv g(0)$ and AF requires $b>0$. The value $g=0$ is a trivial fixed point of eq. (2.4) and so we take $g>0$ without loss of generality. For $\mathrm{SU}(2)_{a}$ and $\mathrm{SU}(3)_{c}$ the constant $b$ for the corresponding gauge couplings $g_{a}$ and $g_{s}$ reads, respectively,

$$
b_{a}=\frac{14}{3}, \quad b_{s}=\frac{29}{3}-\Delta,
$$

where $\Delta$ is the positive extra contribution due to the fermions and scalars in the SM sector. In the numerical calculation we will use for definiteness the reference value $\Delta=28 / 3$, which is compatible with known TAF SM extensions [24,68]. The RGE of $y$ is instead

$$
\frac{d y^{2}}{d t}=y^{2}\left(\frac{9 y^{2}}{2}-8 g_{s}^{2}-\frac{9 g_{a}^{2}}{2}\right)
$$

Like for the gauge couplings, the beta-function vanishes at zero coupling, so we take $y>0$ without loss of generality. This equation admits a closed-form solution for any $b_{a}$ and $b_{s}$ [24]. Finally, the RGEs of $\lambda_{1}$ and $\lambda_{2}$ are $\frac{d \lambda_{1}}{d t}=\beta_{1}$, and $\frac{d \lambda_{2}}{d t}=\beta_{2}$, where [24]

$$
\beta_{1}\left(g_{a}, y, \lambda\right)=\frac{9}{2} g_{a}^{4}+\lambda_{1}\left(8 \lambda_{2}+6 y^{2}-12 g_{a}^{2}\right)+14 \lambda_{1}^{2}+8 \lambda_{2}^{2}-3 y^{4}
$$

and

$$
\beta_{2}\left(g_{a}, y, \lambda\right)=\frac{3}{2} g_{a}^{4}+\lambda_{2}\left(12 \lambda_{1}+6 y^{2}-12 g_{a}^{2}\right)+6 \lambda_{2}^{2}+\frac{3}{2} y^{4} .
$$

Note that $\lambda_{1}=0$ and $\lambda_{2}=0$ generically are not zeros of $\beta_{1}$ and $\beta_{2}$ and, therefore, the quartic couplings, or some combinations thereof, could a priori change sign and pass through zero during the RG evolution.

In [24] the system of equations (2.4)-(2.8) was solved and it was found that for any initial condition for the gauge couplings there is one and only one TAF solution satisfying the stability conditions in (2.3) at high-field values. For such solution both $y$ and $\lambda_{i}$ are IR attractive and are, therefore, predicted at low energies [24]. We will pick up this TAF solution from now on as the high-field stability conditions in (2.3) are necessary to have a viable setup.

\section{Quantum generation of $f_{a}$}

The RGEs dictate that the couplings run with energy. The conditions in (2.3) are necessary at high energy for high-field stability, but they can be violated in the IR. In the present model we find that, while the first condition is always preserved, the second one is violated at small energy. This leads to spontaneous symmetry breaking of $\mathrm{U}(1)_{\mathrm{PQ}}$ through the $\mathrm{CW}$ mechanism [30], because at the energy scale $\mu_{\mathrm{PQ}}$ where $\lambda \equiv \lambda_{1}+\lambda_{2}=0$ the effective potential develops a flat direction (corresponding to $A=A^{\dagger}$, such that the two terms in the potential (2.2) become equal: $\left.\operatorname{Tr}\left(A^{\dagger} A\right)=\operatorname{Tr}(A A)\right)$. We interpret $\mu_{\mathrm{PQ}}$ as the PQ scale. On the flat direction $A=A^{\dagger}$ the three components $A_{k}$ of $A$ along the Pauli matrices, $A=A_{k} \sigma^{k} / 2$, can always be transformed through an element of $\mathrm{SU}(2)_{a}$ in a way that only 
one of these components is not vanishing and positive. We call this non-zero component $\phi$. For example, a possible choice for $\phi$ is $\phi=\left|\operatorname{Re}\left(A_{1}\right)\right|$. In [24] the case in which the explicit mass $m$ is larger than $\mu_{\mathrm{PQ}}$ was considered. Here we focus instead on the opposite case $m \ll \mu_{\mathrm{PQ}}$, so the PQ symmetry breaking is entirely driven by the CW mechanism.

A non-vanishing value of $\phi$ breaks $\mathrm{SU}(2)_{a}$ down to a residual Abelian group $\mathrm{U}(1)_{a}$ leading to a massless spin-1 particle (a dark photon), two spin-1 particles with equal mass

$$
M_{V}(\phi)=g_{a} \phi
$$

(which can be described by one complex vector field), two degenerate Dirac fermions with mass

$$
M_{Q}(\phi)=y \phi / 2
$$

two scalars with squared mass

$$
M_{S}^{2}(\phi)=\left(\lambda_{1}-\lambda_{2}\right) \phi^{2}
$$

and two massless scalars (one is the axion, which as usual acquires a mass through quantum correction, and the other one corresponds to the flat direction). Note that $M_{S}^{2} \geq 0$ when $\lambda_{1} \geq \lambda_{2}$, which turns out to be satisfied at all scales, from the TAF requirement [24].

At one-loop the quantum potential at zero temperature along the flat direction is given by

$$
V_{\mathrm{CW}}(\phi)=V_{0}(\phi)+V_{1}(\phi),
$$

where $V_{0}$ is the tree-level potential, where $\lambda$ is evaluated at the renormalization scale $\mu=\mu_{0} \exp \left(8 \pi^{2} t\right)$

$$
V_{0}(\phi)=\frac{\lambda(t)}{4} \phi^{4}
$$

and $V_{1}$ is the quantum one-loop correction

$$
V_{1}(\phi)=\sum_{b} \frac{n_{b} M_{b}(\phi)^{4}}{4(4 \pi)^{2}}\left(\ln \left(\frac{M_{b}^{2}(\phi)}{\mu^{2}}\right)-a_{b}\right)-\sum_{f} \frac{n_{f} M_{f}(\phi)^{4}}{4(4 \pi)^{2}}\left(\ln \left(\frac{M_{f}^{2}(\phi)}{\mu^{2}}\right)-a_{f}\right) .
$$

In this expression the sum over $b$ runs over all bosons (with number of degrees of freedom $n_{b}$ ), that over $f$ runs over all fermions (with number of degrees of freedom $n_{f}$ ), $M_{b, f}(\phi)$ are the corresponding background-dependent masses (which, for our model, are given in eqs. (3.1), (3.2) and (3.3)) and $a_{b}$ and $a_{f}$ are renormalization-scheme dependent quantities. It is understood that the coupling constants in $V_{1}$ are evaluated at the same renormalization scale, $\mu$. This part of the potential can be computed explicitly by using the backgrounddependent masses given above. Setting $\mu=\mu_{\mathrm{PQ}}$, where $\lambda$ vanishes, leads to

$$
V_{\mathrm{CW}}(\phi)=\frac{\bar{\beta}}{4}\left(\ln \left(\frac{\phi}{f_{a}}\right)-\frac{1}{4}\right) \phi^{4},
$$

where $\bar{\beta}$ is the beta-function of $\lambda$ evaluated at $\mu_{\mathrm{PQ}}$, namely

$$
\bar{\beta} \equiv\left[\mu \frac{d \lambda}{d \mu}\right]_{\mu=\mu_{\mathrm{PQ}}}
$$




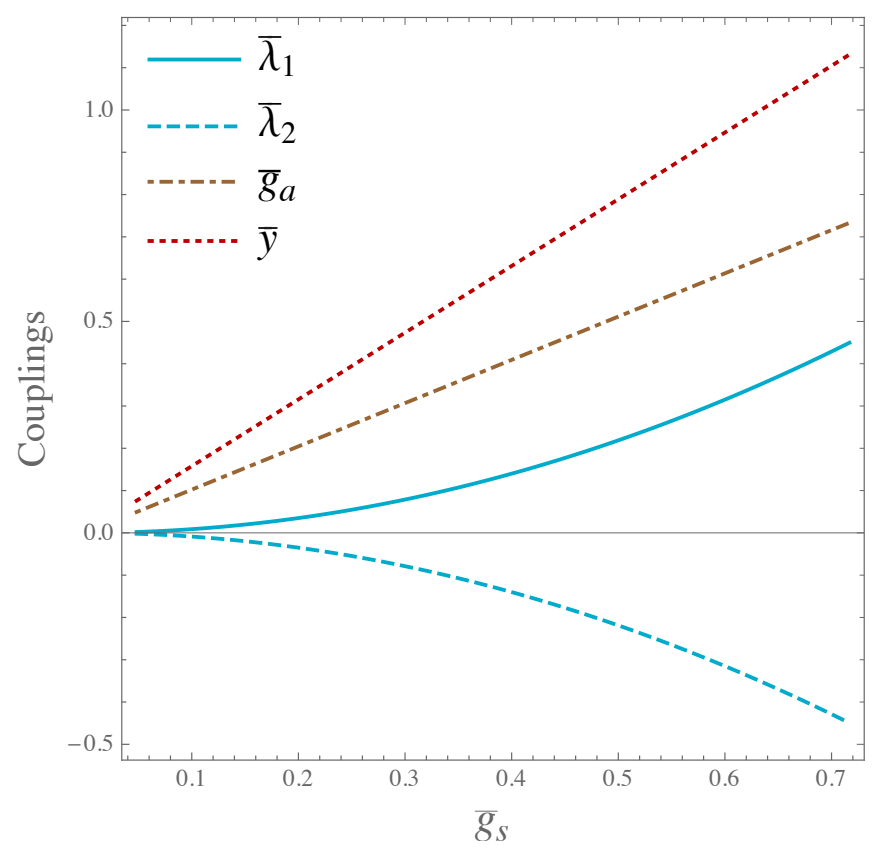

Figure 1. The couplings of the model as functions of the QCD gauge coupling at the PQ scale.

and the scale $f_{a}$ has been introduced in a way that the CW potential, eq. (3.7), has its stationary point at $\phi=f_{a}$. We conventionally choose a renormalization scheme such that $f_{a}=\exp (-1 / 4) \mu_{\mathrm{PQ}}$. When $\bar{\beta}>0$ the stationary point at $\phi=f_{a}$ corresponds to a minimum. We have numerically verified the positivity of $\bar{\beta}$ in our model. Then $\mathrm{U}(1)_{\mathrm{PQ}}$ is spontaneously broken and $\phi$ acquires the VEV $\langle\phi\rangle=f_{a}$ and a mass squared $M_{\phi}=\sqrt{\bar{\beta}} f_{a}$. The energy scale $f_{a}$ is, therefore, identified with the PQ symmetry breaking scale. The remaining mass spectrum besides $M_{\phi}$ corresponds to the axion, the dark photon and the tree-level masses obtained by setting $\phi=f_{a}$ in eqs. (3.1), (3.2) and (3.3). Since $f_{a}$ above the scale of $10^{8} \mathrm{GeV}$ and the mass of the extra-quarks $M_{Q}\left(f_{a}\right)$ is around $f_{a}$, the dark photon satisfies all present bounds, including those of cosmological nature [24].

Note that the arbitrariness of $\mu_{0}$ tells us that $\mu_{\mathrm{PQ}}$ (and thus $f_{a}$ ) is a free parameter. The TAF axion sector features only another free parameter, which can be taken to be $\bar{g}_{s} \equiv g_{s}\left(t_{\mathrm{PQ}}\right)$ (in this paper a bar indicates that a generic coupling is evaluated at $t=$ $\left.t_{\mathrm{PQ}} \equiv \ln \left(\mu_{\mathrm{PQ}}^{2} / \mu_{0}^{2}\right) /(4 \pi)^{2}\right)$. Indeed, once the gauge couplings are chosen at $\mu=\mu_{\mathrm{PQ}}$ the other couplings $\bar{y}, \bar{\lambda}_{1}$ and $\bar{\lambda}_{2}$ are predicted [24] and one must consider a particular IR value of one of the gauge couplings, say $\bar{g}_{a}$, to enforce $\bar{\lambda}_{1}+\bar{\lambda}_{2}=0$, namely to have CW symmetry breaking. In figure 1 we give the couplings $\bar{g}_{a}, \bar{y}$ and $\bar{\lambda}_{1}=-\bar{\lambda}_{2}$ as functions of $\bar{g}_{s}$ to show that all dimensionless quantities in the axion sector are fixed once $\bar{g}_{s}$ is chosen. As a result, when the PQ symmetry is broken à la $\mathrm{CW}$ one also obtains a prediction for the mass of the extra complex vector field (in addition to the predictions of the extra scalar and fermion masses [24]) once $f_{a}$ and $\bar{g}_{s}$ are chosen. Therefore, we explicitly see that the $\mathrm{CW}$ mechanism to break $\mathrm{U}(1)_{\mathrm{PQ}}$ leads to a more predictive framework than the symmetry breaking mechanism based on the explicit mass term $m^{2} \operatorname{Tr}\left(A^{\dagger} A\right)$. 


\section{Peccei-Quinn phase transition}

In order to investigate the nature of the Peccei-Quinn phase transition we take into account thermal corrections as well as quantum corrections. We consider the one-loop effective potential

$$
V_{\text {eff }}(\phi, T) \equiv V_{\mathrm{CW}}(\phi)+V_{T}(\phi)+\Lambda_{0},
$$

where the thermal correction $V_{T}$ to the effective potential is given by [72] (see also [73])

$$
V_{T}(\phi)=\frac{T^{4}}{2 \pi^{2}}\left(\sum_{b} n_{b} J_{B}\left(M_{b}^{2}(\phi) / T^{2}\right)-\sum_{f} n_{f} J_{F}\left(M_{f}^{2}(\phi) / T^{2}\right)\right),
$$

with

$$
J_{B, F}(x) \equiv \int_{0}^{\infty} d q q^{2} \ln \left[1 \mp \exp \left(-\sqrt{q^{2}+x}\right)\right],
$$

and we have included in $V_{\text {eff }}(\phi, T)$ a constant term $\Lambda_{0}$ to account for the observed value of the cosmological constant when $\phi$ is at the minimum. It is understood that the coupling constants in $V_{T}$ are evaluated at the same renormalization scale, $\mu$, used in $V_{\mathrm{CW}}$.

Since the background-dependent squared masses are all non-negative $V_{\text {eff }}$ has a vanishing imaginary part. This is due to the fact that $f_{a}$ is generated quantum mechanically rather than through an explicit tachyonic scalar mass, which would unavoidably lead to a concave tree-level potential and thus to a complex effective potential for some field values. Therefore, the CW symmetry breaking supports the validity of perturbation theory: indeed, a non-negligible imaginary part (absent in the CW case) generically signals the breaking of the perturbative expansion. Further comments regarding the approximation used will be given below in this section.

In figure 2 (left plot) we show $V_{\text {eff }}$ as a function of $\phi$ for two values of the temperature: the critical temperature $T_{c}$ and $T=0$. That figure shows that the transition is of first order. Although we use a fixed value of $\bar{g}_{s}$ in that figure, other choices of this parameter lead to the same qualitative situation. In the right plot of figure 2 we give the dimensionless quantity $T_{c} / f_{a}$ as a function of the only dimensionless parameter of the axion sector, $\bar{g}_{s}$.

The absolute minimum of the effective potential is at $\langle\phi\rangle=0$ for $T>T_{c}$, while, for $T<T_{c}$, is at a non-vanishing temperature-dependent value. In the latter case the decay rate per unit volume $\Gamma$ of the false vacuum $\phi=0$ into the true vacuum $\phi=\langle\phi\rangle \neq 0$ can be computed with the formalism of [74-77]:

$$
\Gamma \approx \max \left(T^{4}\left(\frac{S_{3}}{2 \pi T}\right)^{3 / 2} \exp \left(-S_{3} / T\right), \frac{1}{R_{4}^{4}}\left(\frac{S_{4}}{2 \pi}\right)^{2} \exp \left(-S_{4}\right)\right) .
$$

Here $S_{d}$ is the action

$$
S_{d}=\frac{2 \pi^{d / 2}}{\Gamma(d / 2)} \int_{0}^{\infty} d r r^{d-1}\left(\frac{1}{2} \phi^{\prime 2}+V_{\mathrm{eff}}(\phi, T)\right)
$$

evaluated at the $\mathrm{O}(d)$ bounce defined as the solution of the differential problem

$$
\phi^{\prime \prime}+\frac{d-1}{r} \phi^{\prime}=\frac{d V_{\mathrm{eff}}}{d \phi}, \quad \phi^{\prime}(0)=0, \quad \lim _{r \rightarrow \infty} \phi(r)=0
$$



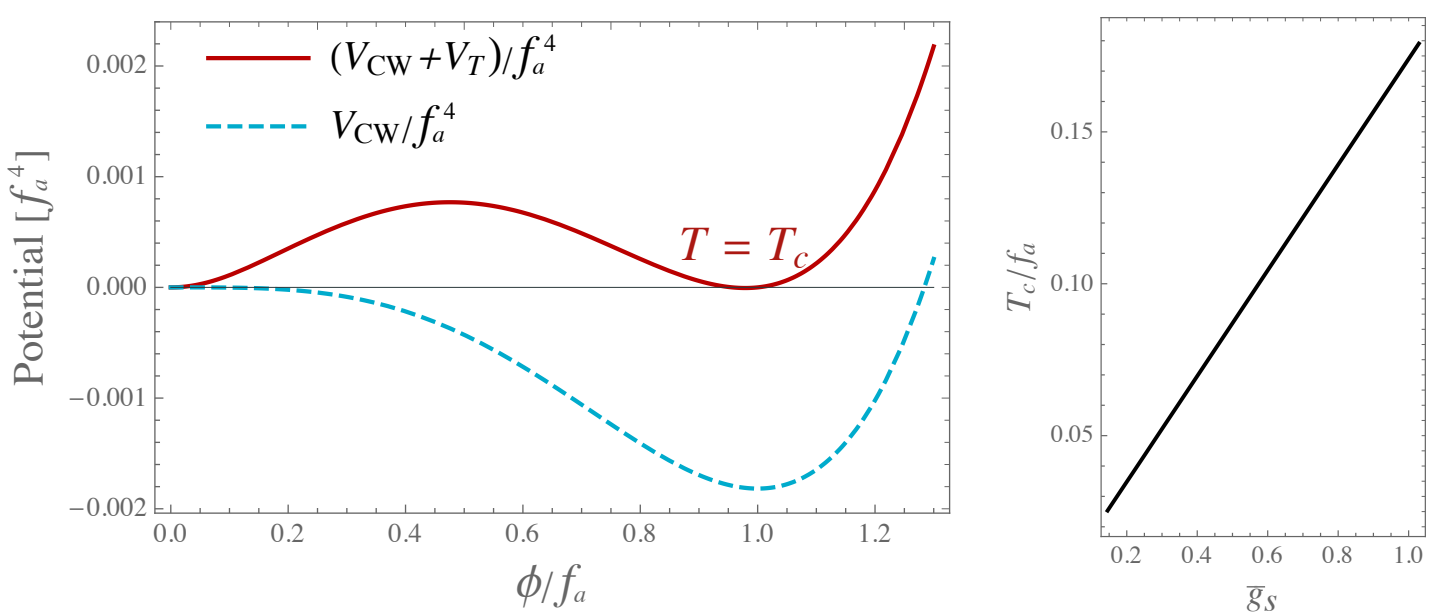

Figure 2. Left plot: the effective potential setting the QCD gauge coupling at the PQ scale to $\bar{g}_{s} \approx 0.91$ and adding a constant such that it vanishes at $\phi=0$. Right plot: the critical temperature $T_{c}$ divided by $f_{a}$ as a function of $\bar{g}_{s}$.

where a prime denotes a derivative with respect to $r$. Also, $R_{4}$ is the size of the $O(4)$ bounce. Note that $S_{d}$ evaluated at the $\mathrm{O}(d)$ bounce can be simplified through the scaling arguments of [78] to obtain

$$
S_{d}=\frac{4 \pi^{d / 2} / \Gamma(d / 2)}{(2-d)} \int_{0}^{\infty} d r r^{d-1} V_{\mathrm{eff}}(\phi, T)
$$

Using the expression above instead of the one in (4.5) makes numerical calculations easier because the derivative of the bounce does not appear in the action.

We numerically checked that $S_{3} / T<S_{4}$, so $\Gamma$ is dominated by the $\mathrm{O}(3)$ bounce. Therefore, the phase transition is essentially due to thermal effects rather than quantum effects. As an example, in figure 3 we give a plot of the $\mathrm{O}(3)$ and $\mathrm{O}(4)$ bounces for representative values of the QCD gauge coupling and the temperature below $T_{c}$.

Some words on the approximation used are now in order. We note that the correction to the two-derivative term in the one-loop effective action is small as long as the temperature is small compared to the field values [79] characterising the bounce solution. We have checked that $T$ is small compared to (few $\%$ of) the relevant field values for all numerical calculations performed in this work. This also implies that we are far from the hightemperature regime for which the perturbative expansion is known to break down [80]. Moreover, note that, generically, the higher-derivative corrections to the one-loop effective action are suppressed when the laplacian applied to the solution of interest (in this case the bounce) is small compared to the background dependent masses times that solution: this follows from the structure of the equations of motion without higher derivatives. Since the largest couplings are of order one in our case, those higher-derivative corrections are small when $\frac{d V_{\text {eff }}}{d \phi}$ is small compared to $\phi^{3}$ in the relevant range of $r$ (where $S_{3} / T$ gets its dominant contribution). We numerically checked that this condition is also satisfied (at the few $\%$ level). Therefore, our one-loop approximation for the effective action is reliable. 


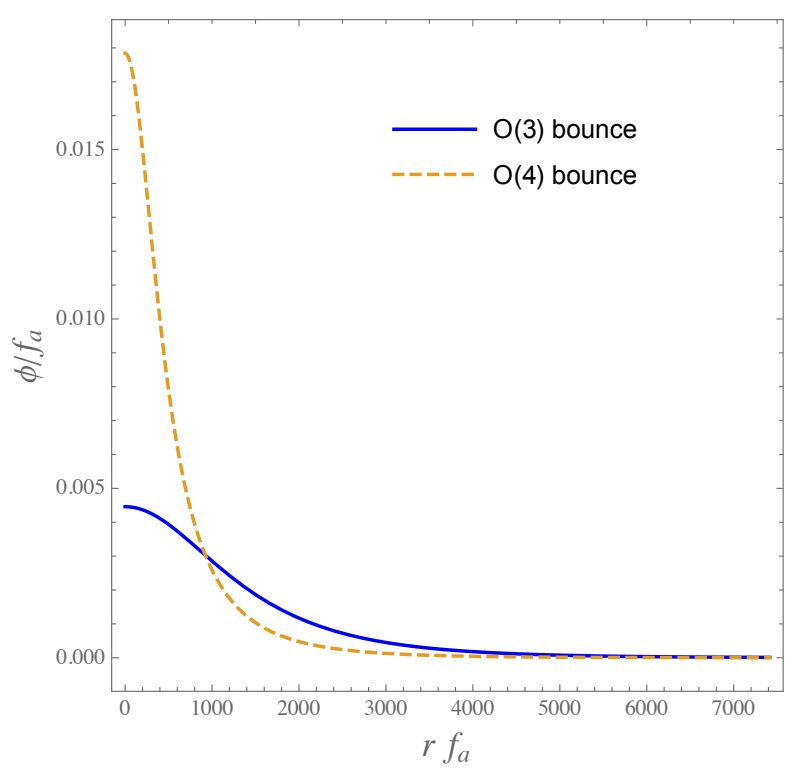

Figure 3. The $\mathrm{O}(\mathrm{d})$-symmetric bounces for $\bar{g}_{s}=0.91$ and $T \approx 4 \times 10^{-3} T_{c} \approx 6.3 \times 10^{-4} f_{a}$.

Also the gravitational corrections to the false vacuum decay are amply negligible in our case. This is because the typical scales of the bounce and the temperature are always below $f_{a}$ (as illustrated in figures 2 and 3 ) and the gravitational corrections are, therefore, suppressed by factors at least as small as $f_{a}^{2} / \bar{M}_{P}^{2}$ [81-85]. The latter quantity is tiny because $f_{a}$ is several orders of magnitude below $\bar{M}_{P}$ in order for the axion not to overproduce DM (see ref. [6] for a review).

Like for other models with CW symmetry breaking [11, 12], we find that when $T$ goes below $T_{c}$ the scalar field $\phi$ is trapped in the false vacuum $\phi=0$ until $T$ is much below $T_{c}$, in other words the universe features a phase of strong supercooling. Then the energy density is dominated by the vacuum energy of $\phi$ and the universe grows exponentially like during inflation but with Hubble rate $H_{I}=\sqrt{\bar{\beta}} f_{a}^{2} /\left(4 \sqrt{3} \bar{M}_{P}\right)$, where $\bar{M}_{P}$ is the reduced Planck mass that is defined in terms of the Planck mass $M_{P}$ by $\bar{M}_{P} \equiv M_{P} / \sqrt{8 \pi}$. The bubbles created are diluted by the expansion of the universe and they cannot collide until $T$ reaches the nucleation temperature $T_{n}$, which corresponds to the temperature when $\Gamma / H_{I}^{4} \sim 1$ or, equivalently, using the fact that the decay is dominated by the $\mathrm{O}(3)$ bounce,

$$
\frac{S_{3}}{T_{n}}-\frac{3}{2} \ln \left(\frac{S_{3} / T_{n}}{2 \pi}\right)=4 \ln \left(\frac{T_{n}}{H_{I}}\right) .
$$

In figure 4 (left plot) we give $T_{n}$ as a function of $\bar{g}_{s}$ for two physically interesting values of the PQ symmetry breaking scale: $f_{a}=10^{11} \mathrm{GeV}$ (for which the axion can account for the whole $\mathrm{DM}$ ) and $f_{a}=10^{9} \mathrm{GeV}$ (for which the contribution of the axion to the DM abundance is negligible). ${ }^{3}$ We find that the equation that determines $T_{n}$ in (4.8) does not always admit a solution: there is a minimal value of the coupling $\bar{g}_{s}$ below which there is

\footnotetext{
${ }^{3}$ For $f_{a}=10^{9} \mathrm{GeV}$ DM can be accounted for by other extra fields among those that are compatible with the TAF principle in the SM sector (see e.g. [23, 67, 68]).
} 

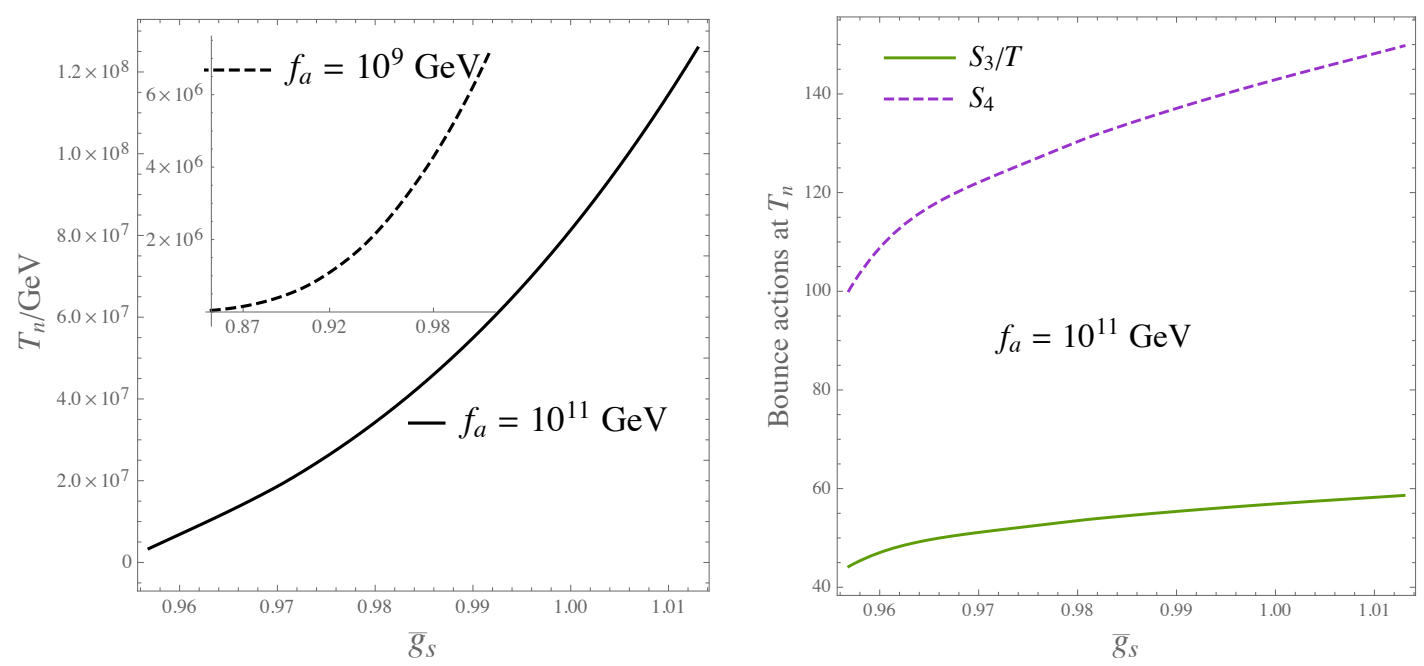

Figure 4. Left plot: the nucleation temperature $T_{n}$ as a function of $\bar{g}_{s}$. Right plot: the bounce actions $S_{3} / T$ and $S_{4}$ evaluated at $T_{n}$ as a function of $\bar{g}_{s}$.

no solution (see the left plot of figure 4). As clear from the left plot of figure 4, when the coupling goes to this minimal value $T_{n}$ becomes very small. By comparing the right plot of figure 2 with the left plot of figure 4 one can see that supercooling generically takes place, namely $T_{n} \ll T_{c}$. In figure 4 we also plot the bounce actions $S_{3} / T$ and $S_{4}$ evaluated at $T_{n}$ as functions of $\bar{g}_{s}$. That plot shows, as mentioned above, that $S_{4}>S_{3} / T$ and so the phase transition is dominated by thermal effects. In the figure we consider, as an example, $f_{a}=10^{11} \mathrm{GeV}$, but the other values of $f_{a}$ lead to the same qualitative behavior. Moreover, an important requirement is that the phase transition completes. The detailed conditions for the true vacuum to fill the entire space were found in [86] and [87]. We have checked that this requirement is satisfied for all numerical calculations performed in this paper.

We also note that strong supercooling and the corresponding inflationary period efficiently dilute the density $n(T)$ of monopoles ${ }^{4}$ due to the breaking $\mathrm{SU}(2)_{a} \rightarrow \mathrm{U}(1)_{a}$. In a strong first-order phase transition monopoles may be created by bubble collisions and well-known estimates $[90,91]$ lead to

$$
\frac{n\left(T_{n}\right)}{T_{n}^{3}} \gtrsim p\left(\frac{T_{n}}{C M_{P}}\right)^{3}
$$

where $p$ is the probability that the scalar field configuration is topologically non trivial, $C=$ $0.6 / \sqrt{g_{*}\left(T_{n}\right)}$ and $g_{*}(T)$ is the effective number of relativistic species in thermal equilibrium at temperature $T$. Even for $p \approx 1$, setting $g_{*}\left(T_{n}\right)$ of order $10^{2}$ (a realistic setup given the existing TAF SM sectors) we find that the theoretical bound in (4.9) is amply compatible (unlike in grand unified theories without inflation) with the bound coming from the fact that the mass density of monopoles must not exceed the limit on the total mass density imposed by the observed Hubble constant and deceleration parameter [90, 91]. Indeed, the

\footnotetext{
${ }^{4}$ See also refs. $[88,89]$ for a discussion on monopole production in the absence of supercooling.
} 
latter bound is around $n\left(T_{0}\right) / T_{0}^{3} \lesssim 10 \mathrm{eV} / M_{m}$, where $T_{0}$ is today's temperature, $M_{m}$ is the monopole mass and $M_{m} \sim 4 \pi f_{a} / \bar{g}_{a}, n\left(T_{0}\right) / T_{0}^{3} \lesssim n\left(T_{n}\right) / T_{n}^{3}$ and the window $10^{8} \mathrm{GeV} \lesssim$ $f_{a} \lesssim 10^{12} \mathrm{GeV}$ have been used.

The strength of the phase transition is measured, as usual, by the parameter $\alpha$ defined as the ratio between

$$
\rho\left(T_{n}\right) \equiv\left[\frac{T}{4} \frac{d}{d T} \Delta V_{\mathrm{eff}}(\langle\phi\rangle, T)-\Delta V_{\mathrm{eff}}(\langle\phi\rangle, T)\right]_{T=T_{n}},
$$

where $\Delta V_{\text {eff }}(\langle\phi\rangle, T) \equiv V_{\text {eff }}(\langle\phi\rangle, T)-V_{\text {eff }}(0, T)$, and the energy density of the thermal plasma (see $[92,93]$ for more details). So

$$
\alpha \equiv \frac{30 \rho\left(T_{n}\right)}{\pi^{2} g_{*}\left(T_{n}\right) T_{n}^{4}}
$$

We find a very strong phase transition with $\alpha$ exceeding one by several orders of magnitude.

At the end of supercooling the universe should be reheated. This occurs in general thanks to the unavoidable coupling between the axion sector and the SM sector due to gluons. The field $\phi$ couples at one loop to gluons through the extra quarks $q$ and $\bar{q}$ so one gets an effective interaction

$$
O_{\mathrm{eff}} \sim \frac{\bar{y} \bar{g}_{s}^{2}}{(4 \pi)^{2}} \phi G_{\mu \nu} G^{\mu \nu} / M_{Q}
$$

where $G_{\mu \nu}$ is the gluon field strength. This leads to the following rate of the decay of $\phi$ into two gluons

$$
\Gamma_{\phi \rightarrow g g} \sim \frac{\bar{y}^{2} \bar{g}_{s}^{4} M_{\phi}^{3}}{(4 \pi)^{5} M_{Q}^{2}} .
$$

We find $\Gamma_{\phi \rightarrow g g} \gg H_{I}$ so the reheating is approximately instantaneous.

The reheating temperature due to this channel may be computed through

$$
T_{\mathrm{RH}}=\left(\frac{45 \Gamma_{\phi \rightarrow g g}^{2} \bar{M}_{P}^{2}}{4 \pi^{3} g_{*}\left(T_{\mathrm{RH}}\right)}\right)^{1 / 4} .
$$

But this formula is only valid if the radiation energy density $\rho_{R}$ does not exceed the vacuum energy density $\rho_{\text {vac }}$ due to $\phi$ (because $\rho_{\text {vac }}$ represents the full energy budget of the system). If this condition is not satisfied we determine $T_{\mathrm{RH}}$ as the maximal temperature compatible with $\rho_{R} \leq \rho_{\text {vac }}$, leading to

$$
T_{\mathrm{RH}}^{4} \approx \frac{15 \bar{\beta} f_{a}^{4}}{8 \pi^{2} g_{*}}
$$

Our estimate of $T_{\mathrm{RH}}$ agrees to very good accuracy with previous determinations [11]. We find a very high $T_{\mathrm{RH}}$. For example, setting $g_{*} \sim 10^{2}$ and $\left\{\bar{g}_{s}, f_{a}\right\} \approx\left\{0.91,1.2 \times 10^{9} \mathrm{GeV}\right\}$ we obtain $T_{\mathrm{RH}} \sim 10^{8} \mathrm{GeV}$, while for $\left\{\bar{g}_{s}, f_{a}\right\} \approx\left\{0.97,10^{11} \mathrm{GeV}\right\}$ we obtain $T_{\mathrm{RH}} \sim 10^{10} \mathrm{GeV}$. Note, nevertheless, that the maximal value of $T_{\mathrm{RH}}$ in (4.15) implies that $T_{\mathrm{RH}}$ is always below $f_{a}$ because $\bar{\beta}$ is a beta function and thus loop suppressed and $g_{*}$ is at least of order $10^{2}$. 


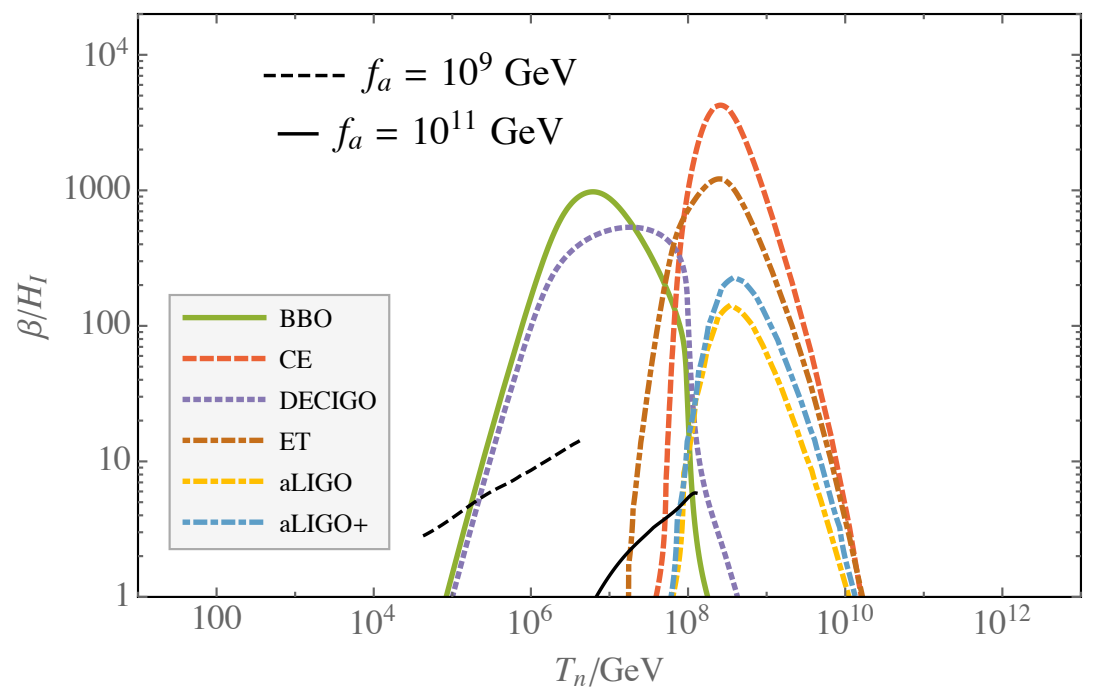

Figure 5. The theoretical prediction in the $\left(T_{n}, \beta / H_{I}\right)$ plane compared with the sensitivity curves of BBO, CE, DECIGO, ET and advanced LIGO (see section 6). The theoretical curves are produced by varying $\bar{g}_{s}$ as in figure 4 . The parameter space enclosed within the experimental curves represents detectable signals.

Finally, another important parameter is the inverse of the duration of the phase transition which, following ref. [12], we define through

$$
\beta \equiv\left[\frac{1}{\Gamma} \frac{d \Gamma}{d t}\right]_{T_{n}} .
$$

This quantity, for fast reheating, can be computed with the formula [12]

$$
\frac{\beta}{H_{I}} \approx\left[T \frac{d}{d T}\left(S_{3} / T\right)-4\right]_{T=T_{n}},
$$

where the term -4 is due to the fact that $\Gamma \propto T^{4}$, because, as we checked, the phase transition is dominated by thermal effects and the extra temperature dependence of $\left(\frac{S_{3}}{2 \pi T}\right)^{3 / 2}$ in (4.4) can be neglected because only logarithmic in the CW case. For the reference values $\left\{\bar{g}_{s}, f_{a}\right\} \approx\left\{0.91,1.2 \times 10^{9} \mathrm{GeV}\right\}$ and $\left\{\bar{g}_{s}, f_{a}\right\} \approx\left\{0.97,10^{11} \mathrm{GeV}\right\}$ we obtain $\beta / H_{I} \approx 7$ and $\beta / H_{I} \approx 2$, respectively. The quantity $\beta / H_{I}$ for other values of $\left\{\bar{g}_{s}, f_{a}\right\}$ is given in figure 5 . The relatively small values of $\beta / H_{I}$ that we obtain indicate a fairly long phase transition. In the same plot we also compare these theoretical values of $\beta / H_{I}$ with the experimental sensitivities (see section 6).

We conclude this section by mentioning that the SM sector might also feature further strong first-order phase transitions. This is due to the fact that the SM gauge group must be extended in order to satisfy the TAF requirement and, therefore, additional symmetry breaking patterns are present. We note, however, that it is possible and natural to expect these further phase transitions at temperatures somewhat below the $\mathrm{TeV}$ or $10 \mathrm{TeV}$ scales because the corresponding new physics can be at those energies (see e.g. [68]). The typical temperatures of the PQ phase transition is instead much bigger as shown in figure 4 (left plot). 


\section{Gravitational waves}

When the temperature drops below $T_{n}$ GWs are produced. The dominant source of GWs are bubble collisions that take place in the vacuum. This is because in the era when $T$ reaches $T_{n}$ the energy density is dominated since a long time by the vacuum energy density associated with $\phi$, which leads to an exponential growth of the cosmological scale factor as we have seen. This inflationary behavior as usual dilutes preexisting matter and radiation and, therefore, we neglect the GW production due to turbulence and sound waves in the cosmic fluid ${ }^{5}[10]$.

From [94] (which used, among other things, the results of [95] based on the envelope approximation) we find the following GW spectrum due to vacuum bubble collisions (valid in the presence of supercooling and $\alpha \gg 1$ )

$$
h^{2} \Omega_{\mathrm{GW}}(f) \approx 1.29 \times 10^{-6}\left(\frac{H\left(T_{\mathrm{RH}}\right)}{\beta}\right)^{2}\left(\frac{100}{g_{*}\left(T_{\mathrm{RH}}\right)}\right)^{1 / 3} \frac{3.8\left(f / f_{\text {peak }}\right)^{2.8}}{1+2.8\left(f / f_{\text {peak }}\right)^{3.8}},
$$

where $f_{\text {peak }}$ is the red-shifted frequency peak today and is given by [94]

$$
f_{\text {peak }} \approx 3.79 \times 10^{2} \frac{\beta}{H\left(T_{\mathrm{RH}}\right)} \frac{T_{\mathrm{RH}}}{10^{10} \mathrm{GeV}}\left(\frac{g_{*}\left(T_{\mathrm{RH}}\right)}{100}\right)^{1 / 6} \mathrm{~Hz} .
$$

Being the reheating almost instantaneous we can approximate the Hubble rate at $T_{\mathrm{RH}}$ with its value $H_{I}$. Some progress has been made to compute the GW spectrum beyond the envelope approximation [96-98], but eq. (5.1) remains to date a reasonable and simple approximation for the bubble collisions that take place in the vacuum [97] (what we are mainly interested in).

In figure 6 we give $h^{2} \Omega_{\mathrm{GW}}$ as a function of $f$ for some relevant values of the parameters and setting as an example $g_{*}=2 \times 10^{2}$ : this is a reasonable value given that the SM sector has to be extended to satisfy the TAF requirement. In the same plot we also compare these theoretical findings with the experimental sensitivities of GW detectors (see section 6).

Note that any cosmic source of GW background acts as an extra radiation component and, therefore, modifies the expansion rate of the Universe. This means that it is highly constrained by big-bang nucleosynthesis (BBN) measurements of primordial elements [99-101]. The measurement of the effective number of neutrino species $N_{\text {eff }}$ and the observational abundance of dueterium and helium, gives us the following bound [10]:

$$
\int_{f_{\mathrm{BBN}}}^{f_{\mathrm{UV}}} \frac{d f}{f} h^{2} \Omega_{\mathrm{GW}}(f)<1.3 \times 10^{-6} \frac{N_{\mathrm{eff}}-3.046}{0.234},
$$

where $f_{\mathrm{BBN}} \sim 10^{-11} \mathrm{~Hz}[10]$ (see e.g. [10,99]) and $f_{\mathrm{UV}}$ is some UV cutoff, which in our case can be conservatively taken to be $\Lambda_{G}$, the scale above which gravity is softened. We have explicitly checked that this bound is satisfied by the theoretical curves in figures 6 by carefully taking into account the integration in the left-hand-side of (5.3) and using

\footnotetext{
${ }^{5}$ We explicitly checked that the inclusion of the efficiency factors of ref. [93] gives a subdominant correction in our case.
} 


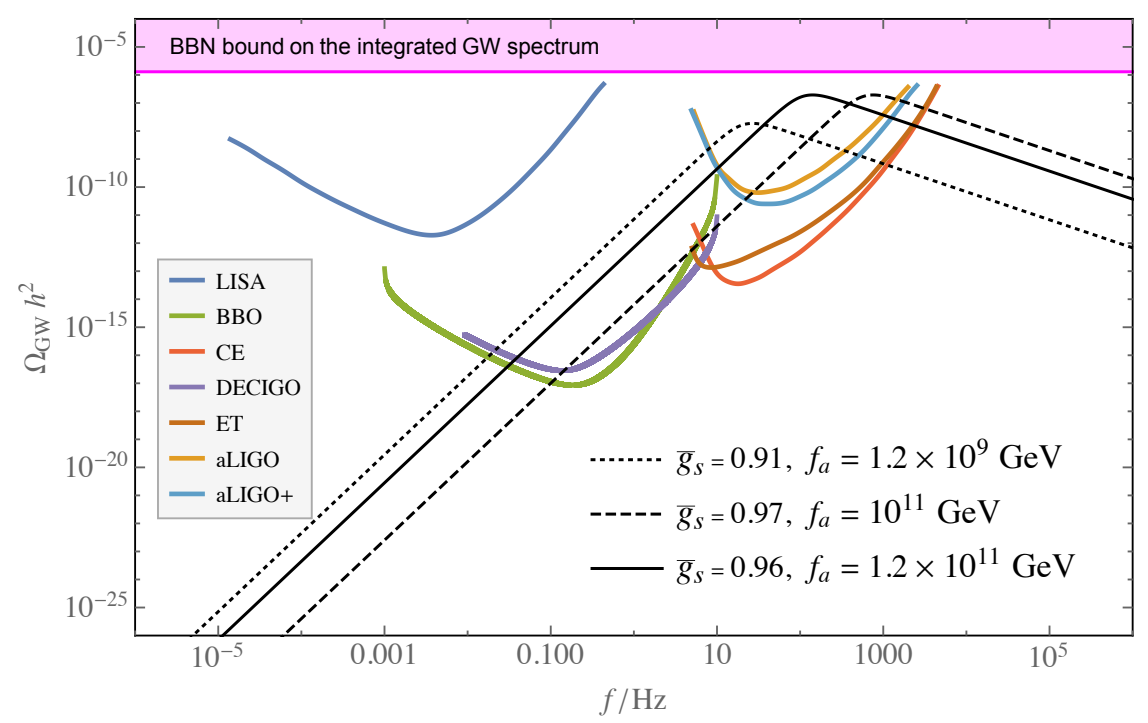

Figure 6. The black lines give the GW amplitude corresponding to the values of the TAF model as mentioned in the figure. The areas above the colored lines correspond to the projected sensitivities for various $\mathrm{GW}$ observatories as detailed in section 6 . The colored region represents the BBN bound on the integrated GW spectrum in (5.3) for the reference upper bound $N_{\text {eff }}=3.28$.

$\Lambda_{G} \ll \bar{M}_{P}$. Note, however, that the bound in (5.3) can also be applied to the nonintegrated GW spectrum unless such spectrum has a very narrow peak (see e.g. [10, 99]), which is not present in any of our results. For simplicity we, therefore, show this bound in figure 6, specifying that it applies to the integrated GW spectrum on the left hand side of (5.3). In doing so we use the reference value $N_{\text {eff }}=3.046+0.234=3.28$, which corresponds to an experimental upper bound on $N_{\text {eff }}$ at $95 \%$ c.l. [10].

Finally, in figure 7 we provide the predictions of the model for the quantities $f_{\text {peak }}$ and $h^{2} \Omega_{\text {peak }} \equiv h^{2} \Omega_{\mathrm{GW}}\left(f_{\text {peak }}\right)$ for allowed values of the parameters of the axion sector $\left(f_{a}\right.$ and $\bar{g}_{s}$ ). We considered values of $f_{a}$ starting from $10^{9} \mathrm{GeV}$ (for which the axion contributes negligibly to DM, but is still compatible with astrophysical bounds) up to values such that the axion accounts for the whole DM. For each fixed value of $f_{a}$ the coupling $\bar{g}_{s}$ is varied above the minimal value discussed in section 4 . In figure 7 the reference value $g_{*}=2 \times 10^{2}$ is again used. As we will see in the next section, these predictions can be efficiently tested through GW observations.

\section{Gravitational wave detectors}

GWs serve as a probe for early universe cosmology, and is particularly important for the era prior to BBN. While inaccessible in collider or in other terrestrial experiments due to the high energy scales involved, the strong first-order phase transition associated with the PQ symmetry breaking induces a stochastic GW source and, therefore, gives us a window to experimentally detect a PQ model. Moreover, observing the particular GW spectrum predicted by the TAF axion model may serve as a first indirect verification of the TAF principle. 


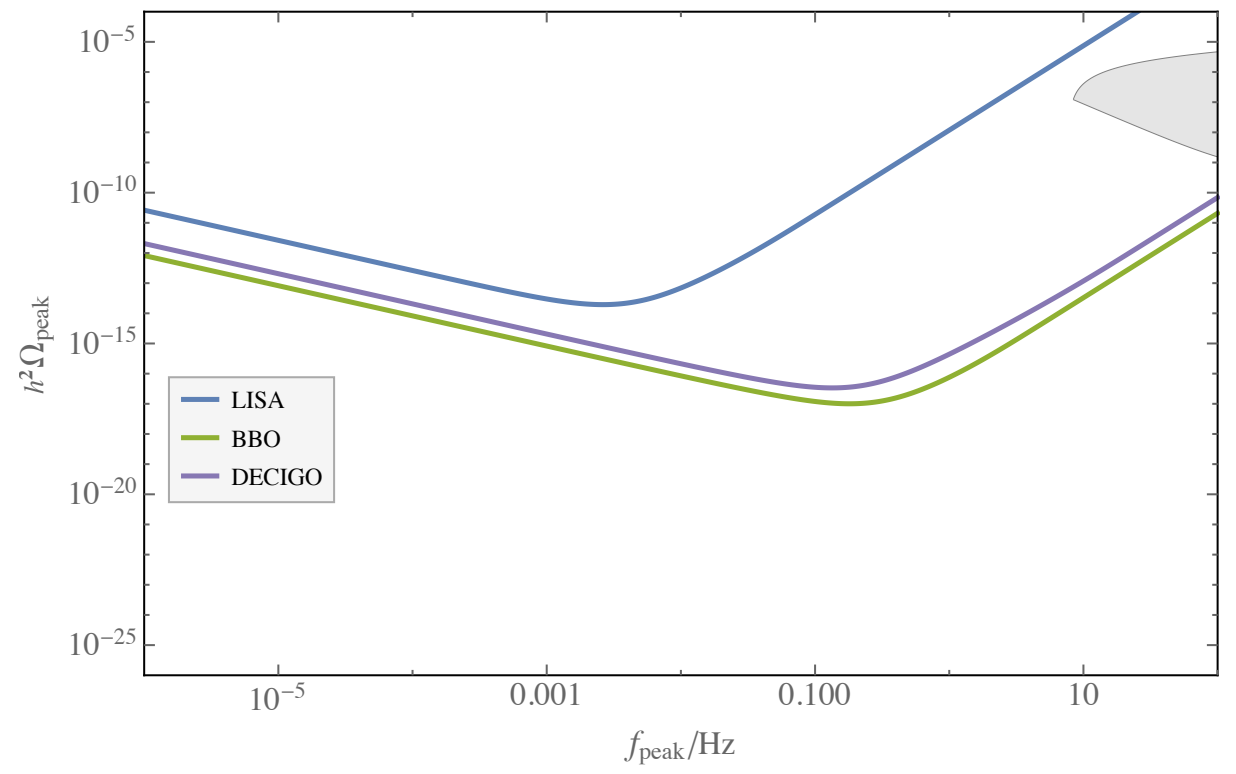

Figure 7. Predictions of the model in the $\left(f_{\text {peak }}, h^{2} \Omega_{\text {peak }}\right)$ plane (grey region) by taking $f_{a} \geq$ $10^{9} \mathrm{GeV}$ (compatibly with astrophysical observations) and $\bar{g}_{s}$ above the minimal values discussed in section 4. Peak-integrated sensitivity curves for LISA, BBO and DECIGO are also shown.

Although there may be other sources of stochastic GWs in the early universe like inflation (refs. [102-105]) or unidentified binary black hole mergers (ref. [106]) the spectrum of GW radiation produced by phase transitions is generically different (see refs. [10, 107] for reviews). For instance, the expected stochastic background from compact binary coalescences, such as binary neutron stars or black holes, has a different dependence on frequency than the one in (5.1), $\Omega_{\mathrm{GW}} \sim f^{2 / 3}$ [108]. Various limits on astrophysical sources contributing as stochastic GW background exist [109]. In 2015, when the GW event (named GW150914) from binary back hole mergers was observed $[9,110]$ and its contribution reanalyzed as a source of stochastic background (with $90 \%$ C.L. statistical uncertainty, propagated from the local rate measurement, on the total background) the event was found to be in the frequency range of $0.01 \mathrm{~Hz}-100 \mathrm{~Hz}$, and of amplitude within the reach of advanced LIGO (see ref. [106] for details). Such range overlaps with the one of the PQ phase transition in the TAF axion model. This, however, is not a problem: due to its weaker frequency dependence, any multiple network of GW detectors, like LIGO [111, 112], VIRGO [113], GEO600 [114], KAGRA [115], and LIGO-India [116], will be able to separate the astrophysical signal from other sources, like the one from a cosmological PQ phase transition or other events taking place after cosmic inflation. In the TAF axion scenarios, future GW detectors will be able to probe the model as we will discuss in detail below.

All experiments to detect GWs has strain noise power spectrum $\left(\Omega_{\text {noise }}=\right.$ $2 \pi^{2} f^{3} S_{\text {noise }} /\left(3 H_{0}^{2}\right)$, where $H_{0}$ is the Hubble parameter in the present Universe, see e.g. [117]). The quantity $S_{\text {noise }}(f)$ consists of intrinsic noise from the instrument as well as other astrophysical confusion noise $S_{\text {noise }}(f)=S_{\text {ins }}(f)+S_{\text {gcn }}(f)$ (see ref. [118] for details) 
that varies from detector to detector. This leads to a signal-to-noise ratio $\mathcal{R}$ given by [119]

$$
\mathcal{R}^{2}=N t_{\mathrm{obs}} \int_{f_{\min }}^{f_{\max }} d f\left[\frac{\Omega_{\text {signal }}(f)}{\Omega_{\text {noise }}(f)}\right]^{2},
$$

where $t_{\mathrm{obs}}$ denotes the experiment's observing time, $N=1(N=2)$ for experiments that perform an auto (cross) correlation measurement of the stochastic gravitational wave background and $f_{\min }$ and $f_{\max }$ are the minimum and maximum frequency accessible to the detector, respectively. The GW spectrum can be detected by ground-based interferometers; these include advanced LIGO in Hanford and Livingston [111, 112], Cosmic Explorer (CE) [120, 121], Einstein Telescope (ET) [122-124]). Moreover, additional information on the GW spectrum can be obtained through space-based interferometers (BBO [125-127], DECIGO [128, 129], and LISA [130]).

There are astrophysical sources contributing as confusion noise (see e.g. [132]) but its impact becomes insignificant with time as one is able to subtract this due to information from the individual foreground sources, which increases in number. However, we consider no foreground contamination, therefore, the results can be treated as an upper limit of the experimental reach possible in future.

In figure 6, we depict the predicted GW spectra for some benchmark points along with the projected sensitivities of current and future interferometer experiments $[120,122,127,130-136]$. The benchmark points in figure 6 include physically interesting cases with $f_{a} \sim 10^{9} \mathrm{GeV}$ and $f_{a} \sim 10^{11} \mathrm{GeV}$ for different values of $\bar{g}_{s}$. Each GW projected sensitivity is denoted with corresponding legends. As clear from figure 6, Advanced LIGO, ET, CE, DECIGO and BBO will be potentially able to detect GWs produced by the $\mathrm{PQ}$ phase transition in the TAF axion model.

Figure 5 provides sensitivity plots in the $\left(T_{n}, \beta / H\right)$ plane for various experiments and compare them with the theoretical curves from the TAF axion model. We considered the physically interesting cases $f_{a}=10^{11} \mathrm{GeV}$ and $f_{a}=10^{9} \mathrm{GeV}$. Figure 5 shows that ET, CE, $\mathrm{BBO}$ and DECIGO will be able to test this scenario through measurements of $T_{n}$ and $\beta / H$.

In figure 7 we compare the predictions of the TAF axion model in the $\left(f_{\text {peak }}, h^{2} \Omega_{\text {peak }}\right)$ plane with the peak-integrated sensitivity curves for LISA, BBO and DECIGO. These curves are drawn following the method explained in ref. [117]. Looking at figure 7 one can clearly see that BBO and DECIGO have the potential to test the allowed parameter space of the TAF axion model.

Finally, we conclude this section by noting that the possible (if any) strong first-order phase transitions due to the TAF SM sector might also produce detectable GWs. The combination of various planned experiments, which have different sensitivity ranges, can help in distinguishing the PQ phase transition from the possible phase transitions due to TAF SM sector. We do not enter the discussion of these SM phase transitions as highly dependent on the specific SM sector one considers. We leave such specific analysis to future work. 


\section{Conclusions}

The detection of GWs and many upcoming GWs detectors have recently reinforced the interest in phase transitions predicted by particle physics models. In this paper we have studied the PQ phase transition and the corresponding spectrum of GWs in a QCD axion model where all couplings flow to zero in the infinite energy limit (TAF property) and the PQ symmetry breaking scale $f_{a}$ is generated quantum mechanically through the CW mechanism. This fundamental (i.e. UV complete) model features an extra gauge group $\mathrm{SU}(2)_{a}$, which is spontaneously broken to an Abelian $\mathrm{U}(1)_{a}$ subgroup. The low-energy spectrum, therefore, includes a dark photon, which has previously been shown to be compatible with current bounds from particle physics experiments and cosmology [24]. This TAF QCD axion model is highly predictive; indeed, the axion sector has only one independent dimensionful quantity, $f_{a}$, and one independent dimensionless parameter, $\bar{g}_{s}$. Therefore, the masses of the particles in the axion sector are all predicted in terms of these two parameters.

We have found that this model features a first-order PQ phase transition, which is very strong. The presence of only few adjustable parameters results in interesting predictions regarding the main quantities associated with the phase transition, $T_{n}, \beta / H_{I}$, etc, mainly summarized in the left plot of figure 4 and in figure 5. We have shown that, like in previous effective axion models with CW PQ symmetry breaking, the phase transition is characterized by a period of strong supercooling, $T_{n} \ll T_{c}$, when the universe inflated. Thanks to this period the monopole density associated with the breaking $\mathrm{SU}(2)_{a} \rightarrow \mathrm{U}(1)_{a}$ is efficiently diluted. Reheating then generically occurs via the unavoidable couplings between the SM and the axion sector due to gluons, which guarantee a rather large reheating temperature. For $\bar{g}_{s}$ of order 1 , the model predicts values of $T_{n}$ and $\beta / H_{I}$ that are within the reach of future GW detectors, such as ET, CE, DECIGO and BBO (see figure 5).

The key theoretical tool, which we have used to obtain these results regarding the first-order phase transition, is the calculation of the bounce solutions associated with the tunnelling from the PQ symmetric configuration to the PQ breaking vacuum. Within this formalism we have also checked that the phase transition is mainly due to thermal effects rather than quantum effects: the action of the $\mathrm{O}(3)$-symmetric bounce divided by the temperature is always much smaller than the action of the $\mathrm{O}(4)$-symmetric one.

Finally, the predictivity of the model also interestingly leads to a rigid dependence of the GWs spectrum produced by the PQ phase transition on $f_{a}$ and $\bar{g}_{s}$. We have compared this theoretical spectrum with the sensitivities of several future detectors such as ET, CE, DECIGO, BBO and advanced LIGO (see figure 6 and 7), finding conclusively that these experiments will be able to test the fundamental QCD axion model.

We believe that the precision that GW astronomy promises due to the planned worldwide network of GW detectors can make the dream of testing high-scale and fundamental BSM scenarios of UV-completion a reality in near future.

\section{Acknowledgments}

We thank M. Bianchi, A. Dasgupta, R. Frezzotti, S. Hajkarim, N. Tantalo and G. White for useful discussions. 
Open Access. This article is distributed under the terms of the Creative Commons Attribution License (CC-BY 4.0), which permits any use, distribution and reproduction in any medium, provided the original author(s) and source are credited.

\section{References}

[1] R.D. Peccei and H.R. Quinn, CP Conservation in the Presence of Instantons, Phys. Rev. Lett. 38 (1977) 1440 [INSPIRE].

[2] S. Weinberg, A New Light Boson?, Phys. Rev. Lett. 40 (1978) 223 [InSPIRE].

[3] J. Preskill, M.B. Wise and F. Wilczek, Cosmology of the Invisible Axion, Phys. Lett. B 120 (1983) 127 [INSPIRE].

[4] L.F. Abbott and P. Sikivie, A Cosmological Bound on the Invisible Axion, Phys. Lett. B 120 (1983) 133 [INSPIRE].

[5] M. Dine and W. Fischler, The Not So Harmless Axion, Phys. Lett. B 120 (1983) 137 [INSPIRE].

[6] L. Di Luzio, M. Giannotti, E. Nardi and L. Visinelli, The landscape of QCD axion models, Phys. Rept. 870 (2020) 1 [arXiv: 2003.01100] [INSPIRE].

[7] Planck collaboration, Planck 2015 results. XX. Constraints on inflation, Astron. Astrophys. 594 (2016) A20 [arXiv:1502.02114] [INSPIRE].

[8] Planck collaboration, Planck 2018 results. X. Constraints on inflation, Astron. Astrophys. 641 (2020) A10 [arXiv: 1807.06211] [inSPIRE].

[9] LigO Scientific and Virgo collaborations, Observation of Gravitational Waves from a Binary Black Hole Merger, Phys. Rev. Lett. 116 (2016) 061102 [arXiv:1602.03837] [INSPIRE].

[10] M. Maggiore, Gravitational Waves. Vol. 2: Astrophysics and Cosmology, Oxford University Press, Oxford U.K. (2018).

[11] L. Delle Rose, G. Panico, M. Redi and A. Tesi, Gravitational Waves from Supercool Axions, JHEP 04 (2020) 025 [arXiv: 1912.06139] [INSPIRE].

[12] B. Von Harling, A. Pomarol, O. Pujolàs and F. Rompineve, Peccei-Quinn Phase Transition at LIGO, JHEP 04 (2020) 195 [arXiv:1912.07587] [INSPIRE].

[13] P.S.B. Dev and A. Mazumdar, Probing the Scale of New Physics by Advanced LIGO/VIRGO, Phys. Rev. D 93 (2016) 104001 [arXiv: 1602.04203] [InSPIRE].

[14] D. Croon, R. Houtz and V. Sanz, Dynamical Axions and Gravitational Waves, JHEP 07 (2019) 146 [arXiv: 1904.10967] [INSPIRE].

[15] P.S.B. Dev, F. Ferrer, Y. Zhang and Y. Zhang, Gravitational Waves from First-Order Phase Transition in a Simple Axion-Like Particle Model, JCAP 11 (2019) 006 [arXiv: 1905.00891] [INSPIRE].

[16] C.S. Machado, W. Ratzinger, P. Schwaller and B.A. Stefanek, Gravitational wave probes of axionlike particles, Phys. Rev. D 102 (2020) 075033 [arXiv:1912.01007] [INSPIRE].

[17] D.J. Gross and F. Wilczek, Asymptotically Free Gauge Theories. I, Phys. Rev. D 8 (1973) 3633 [INSPIRE]. 
[18] D.J. Gross and F. Wilczek, Ultraviolet Behavior of Nonabelian Gauge Theories, Phys. Rev. Lett. 30 (1973) 1343 [INSPIRE].

[19] D.J. Gross and F. Wilczek, Asymptotically free gauge theories. 2., Phys. Rev. D 9 (1974) 980 [INSPIRE].

[20] H. Politzer, Reliable Perturbative Results for Strong Interactions?, Phys. Rev. Lett. 30 (1973) 1346 [INSPIRE].

[21] S. Weinberg, Critical Phenomena for Field Theorists, in Understanding the Fundamental Constituents of Matter, A. Zichichi eds., Plenum Press, New York U.S.A. (1977), pg. 1.

[22] S. Weinberg, Ultraviolet divergences in quantum theories of gravitation, in General Relativity: An Einstein Centenary Survey, W. Hawking and W. Israel eds., Cambridge University Press, Cambridge U.K. (1980), pg. 790.

[23] G.F. Giudice, G. Isidori, A. Salvio and A. Strumia, Softened Gravity and the Extension of the Standard Model up to Infinite Energy, JHEP 02 (2015) 137 [arXiv:1412.2769] [INSPIRE].

[24] A. Salvio, A fundamental QCD axion model, Phys. Lett. B 808 (2020) 135686 [arXiv:2003.10446] [INSPIRE].

[25] R. Mann, J. Meffe, F. Sannino, T. Steele, Z.-W. Wang and C. Zhang, Asymptotically Safe Standard Model via Vectorlike Fermions, Phys. Rev. Lett. 119 (2017) 261802 [arXiv: 1707. 02942] [INSPIRE].

[26] S. Abel and F. Sannino, Framework for an asymptotically safe Standard Model via dynamical breaking, Phys. Rev. D 96 (2017) 055021 [arXiv: 1707.06638] [INSPIRE].

[27] G.M. Pelaggi, A.D. Plascencia, A. Salvio, F. Sannino, J. Smirnov and A. Strumia, Asymptotically Safe Standard Model Extensions?, Phys. Rev. D 97 (2018) 095013 [arXiv: 1708.00437] [INSPIRE].

[28] A. Ghoshal, A. Mazumdar, N. Okada and D. Villalba, Stability of infinite derivative Abelian Higgs models, Phys. Rev. D 97 (2018) 076011 [arXiv:1709.09222] [INSPIRE].

[29] A. Ghoshal, Scalar dark matter probes the scale of nonlocality, Int. J. Mod. Phys. A 34 (2019) 1950130 [arXiv:1812.02314] [INSPIRE].

[30] S.R. Coleman and E.J. Weinberg, Radiative Corrections as the Origin of Spontaneous Symmetry Breaking, Phys. Rev. D 7 (1973) 1888 [INSPIRE].

[31] E. Witten, Cosmological Consequences of a Light Higgs Boson, Nucl. Phys. B 177 (1981) 477 [INSPIRE].

[32] J. Jaeckel, V.V. Khoze and M. Spannowsky, Hearing the signal of dark sectors with gravitational wave detectors, Phys. Rev. D 94 (2016) 103519 [arXiv:1602.03901] [INSPIRE].

[33] L. Marzola, A. Racioppi and V. Vaskonen, Phase transition and gravitational wave phenomenology of scalar conformal extensions of the Standard Model, Eur. Phys. J. C 77 (2017) 484 [arXiv: 1704.01034] [INSPIRE].

[34] S. Iso, P.D. Serpico and K. Shimada, QCD-Electroweak First-Order Phase Transition in a Supercooled Universe, Phys. Rev. Lett. 119 (2017) 141301 [arXiv:1704.04955] [INSPIRE].

[35] I. Baldes and C. Garcia-Cely, Strong gravitational radiation from a simple dark matter model, JHEP 05 (2019) 190 [arXiv:1809.01198] [INSPIRE]. 
[36] T. Prokopec, J. Rezacek and B. Świeżewska, Gravitational waves from conformal symmetry breaking, JCAP 02 (2019) 009 [arXiv: 1809.11129] [INSPIRE].

[37] V. Brdar, A.J. Helmboldt and J. Kubo, Gravitational Waves from First-Order Phase Transitions: LIGO as a Window to Unexplored Seesaw Scales, JCAP 02 (2019) 021 [arXiv: 1810.12306] [INSPIRE].

[38] C. Marzo, L. Marzola and V. Vaskonen, Phase transition and vacuum stability in the classically conformal B-L model, Eur. Phys. J. C 79 (2019) 601 [arXiv:1811.11169] [INSPIRE].

[39] A. Mohamadnejad, Gravitational waves from scale-invariant vector dark matter model: Probing below the neutrino-floor, Eur. Phys. J. C 80 (2020) 197 [arXiv:1907.08899] [INSPIRE].

[40] A. Salvio and A. Strumia, Agravity, JHEP 06 (2014) 080 [arXiv:1403.4226] [InSPIRE].

[41] K. Kannike et al., Dynamically Induced Planck Scale and Inflation, JHEP 05 (2015) 065 [arXiv: 1502.01334] [INSPIRE].

[42] A. Salvio, Inflationary Perturbations in No-Scale Theories, Eur. Phys. J. C 77 (2017) 267 [arXiv: 1703.08012] [INSPIRE].

[43] A. Salvio and A. Strumia, Agravity up to infinite energy, Eur. Phys. J. C 78 (2018) 124 [arXiv: 1705. 03896] [INSPIRE].

[44] A. Salvio, Solving the Standard Model Problems in Softened Gravity, Phys. Rev. D 94 (2016) 096007 [arXiv: 1608.01194] [INSPIRE].

[45] A. Salvio, Quadratic Gravity, Front. in Phys. 6 (2018) 77 [arXiv:1804.09944] [INSPIRE].

[46] A. Salvio, Metastability in Quadratic Gravity, Phys. Rev. D 99 (2019) 103507 [arXiv: 1902.09557] [INSPIRE].

[47] A. Salvio, Quasi-Conformal Models and the Early Universe, Eur. Phys. J. C 79 (2019) 750 [arXiv: 1907. 00983] [INSPIRE].

[48] A. Salvio and H. Veermäe, Horizonless ultracompact objects and dark matter in quadratic gravity, JCAP 02 (2020) 018 [arXiv: 1912.13333] [INSPIRE].

[49] T. Biswas, A. Mazumdar and W. Siegel, Bouncing universes in string-inspired gravity, JCAP 03 (2006) 009 [hep-th/0508194] [INSPIRE].

[50] L. Modesto, Super-renormalizable Quantum Gravity, Phys. Rev. D 86 (2012) 044005 [arXiv:1107.2403] [INSPIRE].

[51] T. Biswas, E. Gerwick, T. Koivisto and A. Mazumdar, Towards singularity and ghost free theories of gravity, Phys. Rev. Lett. 108 (2012) 031101 [arXiv:1110.5249] [INSPIRE].

[52] V.P. Frolov, Mass-gap for black hole formation in higher derivative and ghost free gravity, Phys. Rev. Lett. 115 (2015) 051102 [arXiv: 1505.00492] [INSPIRE].

[53] A.S. Koshelev and A. Mazumdar, Do massive compact objects without event horizon exist in infinite derivative gravity?, Phys. Rev. D 96 (2017) 084069 [arXiv:1707.00273] [InSPIRE].

[54] L. Buoninfante, A.S. Koshelev, G. Lambiase, J.a. Marto and A. Mazumdar, Conformally-flat, non-singular static metric in infinite derivative gravity, JCAP 06 (2018) 014 [arXiv: 1804.08195] [INSPIRE]. 
[55] B.L. Giacchini and T. de Paula Netto, Effective delta sources and regularity in higher-derivative and ghost-free gravity, JCAP 07 (2019) 013 [arXiv:1809.05907] [INSPIRE].

[56] L. Buoninfante and A. Mazumdar, Nonlocal star as a blackhole mimicker, Phys. Rev. D 100 (2019) 024031 [arXiv: 1903.01542] [INSPIRE].

[57] L.F. Abbott and M.B. Wise, Wormholes and Global Symmetries, Nucl. Phys. B 325 (1989) 687 [INSPIRE].

[58] S.R. Coleman and K.-M. Lee, Wormholes made without massless matter fields, Nucl. Phys. B 329 (1990) 387 [INSPIRE].

[59] R. Kallosh, A.D. Linde, D.A. Linde and L. Susskind, Gravity and global symmetries, Phys. Rev. D 52 (1995) 912 [hep-th/9502069] [INSPIRE].

[60] A. Hebecker, T. Mikhail and P. Soler, Euclidean wormholes, baby universes, and their impact on particle physics and cosmology, Front. Astron. Space Sci. 5 (2018) 35 [arXiv: 1807.00824] [INSPIRE].

[61] H.M. Georgi, L.J. Hall and M.B. Wise, Grand Unified Models With an Automatic \{Peccei-Quinn\} Symmetry, Nucl. Phys. B 192 (1981) 409 [InSPIRE].

[62] M. Dine and N. Seiberg, String Theory and the Strong CP Problem, Nucl. Phys. B 273 (1986) 109 [INSPIRE].

[63] S.M. Barr and D. Seckel, Planck scale corrections to axion models, Phys. Rev. D 46 (1992) 539 [INSPIRE].

[64] M. Kamionkowski and J. March-Russell, Planck scale physics and the Peccei-Quinn mechanism, Phys. Lett. B 282 (1992) 137 [hep-th/9202003] [INSPIRE].

[65] R. Holman, S.D.H. Hsu, T.W. Kephart, E.W. Kolb, R. Watkins and L.M. Widrow, Solutions to the strong CP problem in a world with gravity, Phys. Lett. B 282 (1992) 132 [hep-ph/9203206] [INSPIRE].

[66] S. Ghigna, M. Lusignoli and M. Roncadelli, Instability of the invisible axion, Phys. Lett. B 283 (1992) 278 [INSPIRE].

[67] B. Holdom, J. Ren and C. Zhang, Stable Asymptotically Free Extensions (SAFEs) of the Standard Model, JHEP 03 (2015) 028 [arXiv: 1412.5540] [INSPIRE].

[68] G.M. Pelaggi, A. Strumia and S. Vignali, Totally asymptotically free trinification, JHEP 08 (2015) 130 [arXiv: 1507.06848] [inSPIRE].

[69] J.E. Kim, Weak Interaction Singlet and Strong CP Invariance, Phys. Rev. Lett. 43 (1979) 103 [INSPIRE].

[70] M.A. Shifman, A.I. Vainshtein and V.I. Zakharov, Can Confinement Ensure Natural CP Invariance of Strong Interactions?, Nucl. Phys. B 166 (1980) 493 [InSPIRE].

[71] A. Salvio, A Simple Motivated Completion of the Standard Model below the Planck Scale: Axions and Right-Handed Neutrinos, Phys. Lett. B 743 (2015) 428 [arXiv:1501.03781] [INSPIRE].

[72] L. Dolan and R. Jackiw, Symmetry Behavior at Finite Temperature, Phys. Rev. D 9 (1974) 3320 [INSPIRE].

[73] M. Quirós, Field theory at finite temperature and phase transitions, Helv. Phys. Acta 67 (1994) 451 [INSPIRE]. 
[74] S.R. Coleman, The Fate of the False Vacuum. 1. Semiclassical Theory, Phys. Rev. D 15 (1977) 2929 [Erratum ibid. 16 (1977) 1248] [INSPIRE].

[75] C.G. Callan Jr. and S.R. Coleman, The Fate of the False Vacuum. 2. First Quantum Corrections, Phys. Rev. D 16 (1977) 1762 [InSPIRE].

[76] A.D. Linde, Fate of the False Vacuum at Finite Temperature: Theory and Applications, Phys. Lett. B 100 (1981) 37 [InSPIRE].

[77] A.D. Linde, Decay of the False Vacuum at Finite Temperature, Nucl. Phys. B 216 (1983) 421 [Erratum ibid. 223 (1983) 544] [INSPIRE].

[78] S.R. Coleman, V. Glaser and A. Martin, Action Minima Among Solutions to a Class of Euclidean Scalar Field Equations, Commun. Math. Phys. 58 (1978) 211 [INSPIRE].

[79] I.G. Moss, D.J. Toms and W.A. Wright, The Effective Action at Finite Temperature, Phys. Rev. D 46 (1992) 1671 [INSPIRE].

[80] S. Weinberg, Gauge and Global Symmetries at High Temperature, Phys. Rev. D 9 (1974) 3357 [INSPIRE].

[81] S.R. Coleman and F. De Luccia, Gravitational Effects on and of Vacuum Decay, Phys. Rev. D 21 (1980) 3305 [INSPIRE].

[82] G. Isidori, V.S. Rychkov, A. Strumia and N. Tetradis, Gravitational corrections to standard model vacuum decay, Phys. Rev. D 77 (2008) 025034 [arXiv:0712.0242] [INSPIRE].

[83] A. Salvio, A. Strumia, N. Tetradis and A. Urbano, On gravitational and thermal corrections to vacuum decay, JHEP 09 (2016) 054 [arXiv: 1608.02555] [INSPIRE].

[84] A. Joti et al., (Higgs) vacuum decay during inflation, JHEP 07 (2017) 058 [arXiv: 1706.00792] [INSPIRE].

[85] T. Markkanen, A. Rajantie and S. Stopyra, Cosmological Aspects of Higgs Vacuum Metastability, Front. Astron. Space Sci. 5 (2018) 40 [arXiv:1809.06923] [INSPIRE].

[86] J. Ellis, M. Lewicki and J.M. No, On the Maximal Strength of a First-Order Electroweak Phase Transition and its Gravitational Wave Signal, JCAP 04 (2019) 003 [arXiv: 1809.08242] [INSPIRE].

[87] J. Ellis, M. Lewicki and J.M. No, Gravitational waves from first-order cosmological phase transitions: lifetime of the sound wave source, JCAP 07 (2020) 050 [arXiv:2003.07360] [INSPIRE].

[88] R. Sato, F. Takahashi and M. Yamada, Unified Origin of Axion and Monopole Dark Matter, and Solution to the Domain-wall Problem, Phys. Rev. D 98 (2018) 043535 [arXiv:1805.10533] [INSPIRE].

[89] C. Chatterjee, T. Higaki and M. Nitta, Note on a solution to domain wall problem with the Lazarides-Shafi mechanism in axion dark matter models, Phys. Rev. D 101 (2020) 075026 [arXiv:1903.11753] [INSPIRE].

[90] J. Preskill, Cosmological Production of Superheavy Magnetic Monopoles, Phys. Rev. Lett. 43 (1979) 1365 [INSPIRE].

[91] J. Preskill, Magnetic monopoles, Ann. Rev. Nucl. Part. Sci. 34 (1984) 461 [inSPIRE].

[92] C. Caprini et al., Detecting gravitational waves from cosmological phase transitions with LISA: an update, JCAP 03 (2020) 024 [arXiv:1910.13125] [INSPIRE]. 
[93] J. Ellis, M. Lewicki, J.M. No and V. Vaskonen, Gravitational wave energy budget in strongly supercooled phase transitions, JCAP 06 (2019) 024 [arXiv: 1903.09642] [INSPIRE].

[94] C. Caprini et al., Science with the space-based interferometer eLISA. II: Gravitational waves from cosmological phase transitions, JCAP 04 (2016) 001 [arXiv:1512.06239] [INSPIRE].

[95] S.J. Huber and T. Konstandin, Gravitational Wave Production by Collisions: More Bubbles, JCAP 09 (2008) 022 [arXiv:0806.1828] [INSPIRE].

[96] R. Jinno and M. Takimoto, Gravitational waves from bubble dynamics: Beyond the Envelope, JCAP 01 (2019) 060 [arXiv:1707.03111] [INSPIRE].

[97] T. Konstandin, Gravitational radiation from a bulk flow model, JCAP 03 (2018) 047 [arXiv: 1712.06869] [INSPIRE].

[98] M. Lewicki and V. Vaskonen, Gravitational wave spectra from strongly supercooled phase transitions, Eur. Phys. J. C 80 (2020) 1003 [arXiv:2007.04967] [InSPIRE].

[99] L.A. Boyle and A. Buonanno, Relating gravitational wave constraints from primordial nucleosynthesis, pulsar timing, laser interferometers, and the CMB: Implications for the early Universe, Phys. Rev. D 78 (2008) 043531 [arXiv:0708.2279] [InSPIRE].

[100] A. Stewart and R. Brandenberger, Observational Constraints on Theories with a Blue Spectrum of Tensor Modes, JCAP 08 (2008) 012 [arXiv:0711.4602] [INSPIRE].

[101] K. Kohri and T. Terada, Semianalytic calculation of gravitational wave spectrum nonlinearly induced from primordial curvature perturbations, Phys. Rev. D 97 (2018) 123532 [arXiv: 1804.08577] [INSPIRE].

[102] R. Easther and E.A. Lim, Stochastic gravitational wave production after inflation, JCAP 04 (2006) 010 [astro-ph/0601617] [INSPIRE].

[103] M.S. Turner, Detectability of inflation produced gravitational waves, Phys. Rev. D 55 (1997) 435 [astro-ph/9607066] [INSPIRE].

[104] S. Kuroyanagi, T. Chiba and T. Takahashi, Probing the Universe through the Stochastic Gravitational Wave Background, JCAP 11 (2018) 038 [arXiv:1807.00786] [INSPIRE].

[105] N. Bartolo et al., Science with the space-based interferometer LISA. IV: Probing inflation with gravitational waves, JCAP 12 (2016) 026 [arXiv:1610.06481] [INSPIRE].

[106] LIGO Scientific and Virgo collaborations, GW150914: Implications for the stochastic gravitational wave background from binary black holes, Phys. Rev. Lett. 116 (2016) 131102 [arXiv: 1602.03847] [INSPIRE].

[107] A. Mazumdar and G. White, Review of cosmic phase transitions: their significance and experimental signatures, Rept. Prog. Phys. 82 (2019) 076901 [arXiv: 1811. 01948] [INSPIRE].

[108] C. Wu, V. Mandic and T. Regimbau, Accessibility of the Gravitational-Wave Background due to Binary Coalescences to Second and Third Generation Gravitational-Wave Detectors, Phys. Rev. D 85 (2012) 104024 [arXiv:1112.1898] [inSPIRE].

[109] LIGO ScIEnTIFIC and VIRGO collaborations, An Upper Limit on the Stochastic Gravitational-Wave Background of Cosmological Origin, Nature 460 (2009) 990 [arXiv: 0910 .5772] [INSPIRE].

[110] LIGO ScIENTIFIC and VIRGO collaborations, GW150914: Implications for the stochastic gravitational wave background from binary black holes, Phys. Rev. Lett. 116 (2016) 131102 [arXiv: 1602.03847] [INSPIRE]. 
[111] LIGO Scientific collaboration, Advanced LIGO, Class. Quant. Grav. 32 (2015) 074001 [arXiv: 1411.4547] [INSPIRE].

[112] LIGO ScIENTIFIC collaboration, Advanced LIGO: The next generation of gravitational wave detectors, Class. Quant. Grav. 27 (2010) 084006 [inSPIRE].

[113] VIRGO collaboration, Advanced Virgo: a second-generation interferometric gravitational wave detector, Class. Quant. Grav. 32 (2015) 024001 [arXiv: 1408. 3978] [INSPIRE].

[114] H. Lück et al., The upgrade of GEO600, J. Phys. Conf. Ser. 228 (2010) 012012 [arXiv: 1004.0339] [INSPIRE].

[115] KAGRA collaboration, Detector configuration of KAGRA: The Japanese cryogenic gravitational-wave detector, Class. Quant. Grav. 29 (2012) 124007 [arXiv:1111.7185] [INSPIRE].

[116] C.S. Unnikrishnan, IndIGO and LIGO-India: Scope and plans for gravitational wave research and precision metrology in India, Int. J. Mod. Phys. D 22 (2013) 1341010 [arXiv: 1510.06059] [INSPIRE].

[117] K. Schmitz, New Sensitivity Curves for Gravitational-Wave Experiments, arXiv:2002.04615 [INSPIRE].

[118] T. Alanne, T. Hugle, M. Platscher and K. Schmitz, A fresh look at the gravitational-wave signal from cosmological phase transitions, JHEP 03 (2020) 004 [arXiv:1909.11356] [INSPIRE].

[119] B. Allen and J.D. Romano, Detecting a stochastic background of gravitational radiation: Signal processing strategies and sensitivities, Phys. Rev. D 59 (1999) 102001 [gr-qc/9710117] [INSPIRE].

[120] LIGO ScIENTIFIC collaboration, Exploring the Sensitivity of Next Generation Gravitational Wave Detectors, Class. Quant. Grav. 34 (2017) 044001 [arXiv: 1607. 08697] [INSPIRE].

[121] D. Reitze et al., Cosmic Explorer: The U.S. Contribution to Gravitational-Wave Astronomy beyond LIGO, Bull. Am. Astron. Soc. 51 (2019) 035 [arXiv:1907.04833] [inSPIRE].

[122] M. Punturo et al., The Einstein Telescope: A third-generation gravitational wave observatory, Class. Quant. Grav. 27 (2010) 194002 [INSPIRE].

[123] S. Hild et al., Sensitivity Studies for Third-Generation Gravitational Wave Observatories, Class. Quant. Grav. 28 (2011) 094013 [arXiv: 1012. 0908] [INSPIRE].

[124] B. Sathyaprakash et al., Scientific Objectives of Einstein Telescope, Class. Quant. Grav. 29 (2012) 124013 [Erratum ibid. 30 (2013) 079501] [arXiv: 1206. 0331] [INSPIRE].

[125] J. Crowder and N.J. Cornish, Beyond LISA: Exploring future gravitational wave missions, Phys. Rev. D 72 (2005) 083005 [gr-qc/0506015] [inSPIRE].

[126] G.M. Harry, P. Fritschel, D.A. Shaddock, W. Folkner and E.S. Phinney, Laser interferometry for the big bang observer, Class. Quant. Grav. 23 (2006) 4887 [Erratum ibid. 23 (2006) 7361] [INSPIRE].

[127] V. Corbin and N.J. Cornish, Detecting the cosmic gravitational wave background with the big bang observer, Class. Quant. Grav. 23 (2006) 2435 [gr-qc/0512039] [INSPIRE].

[128] N. Seto, S. Kawamura and T. Nakamura, Possibility of direct measurement of the acceleration of the universe using $0.1-\mathrm{Hz}$ band laser interferometer gravitational wave antenna in space, Phys. Rev. Lett. 87 (2001) 221103 [astro-ph/0108011] [INSPIRE]. 
[129] S. Kawamura et al., The Japanese space gravitational wave antenna DECIGO, Class. Quant. Grav. 23 (2006) S125 [INSPIRE].

[130] LISA collaboration, Laser Interferometer Space Antenna, arXiv:1702.00786 [INSPIRE].

[131] M. Musha Space gravitational wave detector DECIGO/pre-DECIGO, Proc. SPIE 10562 (2017) 105623T.

[132] T. Robson, N.J. Cornish and C. Liu, The construction and use of LISA sensitivity curves, Class. Quant. Grav. 36 (2019) 105011 [arXiv: 1803. 01944] [INSPIRE].

[133] LIGO ScIENTIFIC collaboration, Gravitational wave astronomy with LIGO and similar detectors in the next decade, arXiv:1904.03187 [INSPIRE].

[134] LIGO ScIEnTIFIC collaboration, Instrument Science White Paper, LIGO DCC-T1400316 (2014).

[135] E. Thrane and J.D. Romano, Sensitivity curves for searches for gravitational-wave backgrounds, Phys. Rev. D 88 (2013) 124032 [arXiv:1310.5300] [INSPIRE].

[136] C.J. Moore, R.H. Cole and C.P.L. Berry, Gravitational-wave sensitivity curves, Class. Quant. Grav. 32 (2015) 015014 [arXiv:1408.0740] [INSPIRE]. 